\title{
AUTO LANDING PROCESS FOR AUTONOMOUS FLYING ROBOT BY USING IMAGE PROCESSING BASED ON EDGE DETECTION
}

\author{
Bahram Lavi Sefidgari ${ }^{1}$ and Sahand Pourhassan Shamchi ${ }^{2}$ \\ ${ }^{1}$ Department of Computer Engineering, EMU, Famagusta, Cyprus \\ Bahram. lavi@emu.edu.tr \\ ${ }^{2}$ Department of Mechanical Engineering, EMU, Famagusta, Cyprus \\ Sahand.pourhassan@hotmail.com
}

\begin{abstract}
In today's technological life, everyone is quite familiar with the importance of security measures in our lives. So in this regard, many attempts have been made by researchers and one of them is flying robots technology. One well-known usage of flying robot, perhaps, is its capability in security and care measurements which made this device extremely practical, not only for its unmanned movement, but also for the unique manoeuvre during flight over the arbitrary areas. In this research, the automatic landing of a flying robot is discussed. The system is based on the frequent interruptions that is sent from main microcontroller to camera module in order to take images; these images have been distinguished by image processing system based on edge detection, after analysing the image the system can tell whether or not to land on the ground. This method shows better performance in terms of precision as well as experimentally.
\end{abstract}

\section{KEYWORDS}

Quadcopter, Flying Robot, Image Processing, Edge Detection, Auto Landing

\section{INTRODUCTION}

Quadcopter, also named as quadrotor helicopter, is a vehicle that moves with electric motors. Basically there are four upward rotors which help quadcopter for any kind of manoeuvres within its flying region. Since the quadcopter is classified as unmanned aerial vehicle (UAV), it is believed that by the increasing demand for autonomous UAVs, quadcopters are going to be developed in autonomous control system. In the last decade, due to the military and security reasons many attempts had been conducted related to this issue $[1,2,3]$.These days, the quadcopter is taken into consideration by many of the robotic researchers regarding its complicated structure. Such tendencies provide a platform for us to find answers to the landing challenges faced by this technology. So by the help of a specified marks or (Signs), device can recognize its landing place. This can be done by aids of image processing to detect and extract the predefined image. 
A common limitation to the quadcopters is the battery life during flight [4], and our homemade quadcopter usually fly about 20 minutes with its on-board battery. With this constraint, the best method for automatic landing is to hire the afore-mentioned technique. Using one extra micro controller and small camera can be helpful to save the battery power.

The aim of this research is to develop a real-time system for detecting and tracking the specific place for landing the quadcopter and in this regard, the edge detection have been applied. The system is implemented experimentally. The main purpose of this work is to employ the quadcopter as a safety and security robot in wide range areas.

\section{BACKGROUND}

Quadcopter itself is not a novel technology; instead, controlling it is considered as a new challenge [5-11]. Basically, the automatic landing in quadcopters is a challenging issue for researchers. This paper will provide a guide by using scientific methods to landing off a quadcopter.

\subsection{Quadcopter Dynamic Model}

Quadcopter robot is controlled by various angular speeds produced from each motor. It has four rotors arranged in cross shape. The front and back rotors are rotating counter of clockwise direction and the left and right rotors are rotating on clockwise side [12]. This structure is shown in figure 1 .

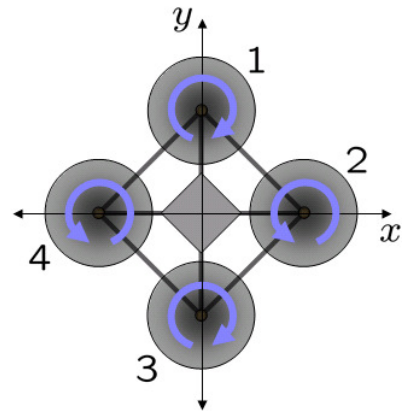

Figure 1. Whole structure of rotations in quadcopter

The quadcopter, which is considered in this work, is classified as a small size of flying robot with limited weight, less than $1 \mathrm{~kg}$. Integration of a homemade flying robot with small camera is required, as well as having an adequate embedded image processing platform. The prototype of implementation with detail is shown in figure. 2 . 


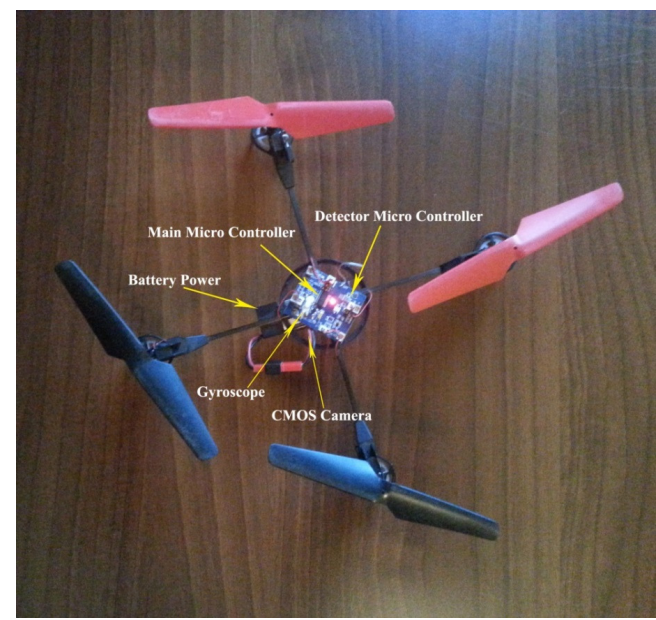

Figure 2. Prototype of quadcopter with detail

The control of quadcopter which was provided by PID controller and coefficients was improved by genetic algorithm $[13,14]$ which was implemented on AVR microcontroller. The execution of the overall system was evaluated in real-time experiments. A small CMOS camera module with photographic array $320 * 240$ was embedded as shown in figure 3 .

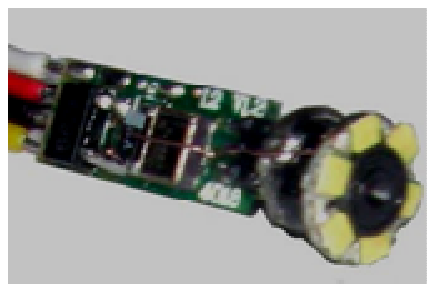

Figure 3. CMOS camera module

\subsection{Image Processing in Quadcopters}

Landing place detection consists of detecting and tracing special shapes which was pre-defined for landing the quadcopter. There are many algorithms that are developed for image processing, such as HOG and LBP [15], Haar wavelets and EOH [16], region covariance matrix [17-18], partial least squares [19], edge detection[20], stereo vision, monocular vision, laser and vision [21-25], sonar and vision, and thermal vision [26-29]. To the best of our knowledge, most of the image processing algorithms can be used in our paper; however, based on our previous experience in edge detection, this algorithm will be employed for detecting special shapes.

\section{LANDing Place Detection Methods on QuAdCoPTer}

\subsection{System Overview}

Landing Detection and tracing system extract the exact place which is defined for landing the vehicle. It recognizes an object from the altitude (approximately up to 10meter) and employs geometric and edge detection algorithms to check whether the object has circular shapes or not. The extraction of circular shapes, which defines our place for landing, could be used instead of high quality image. In this regard, by using interruption emitted from main microcontroller to 
take frequent images and identify landing scope. Figure 4 presents the diagram of proposed system and figure 5 presents the algorithm which is implemented in this paper.

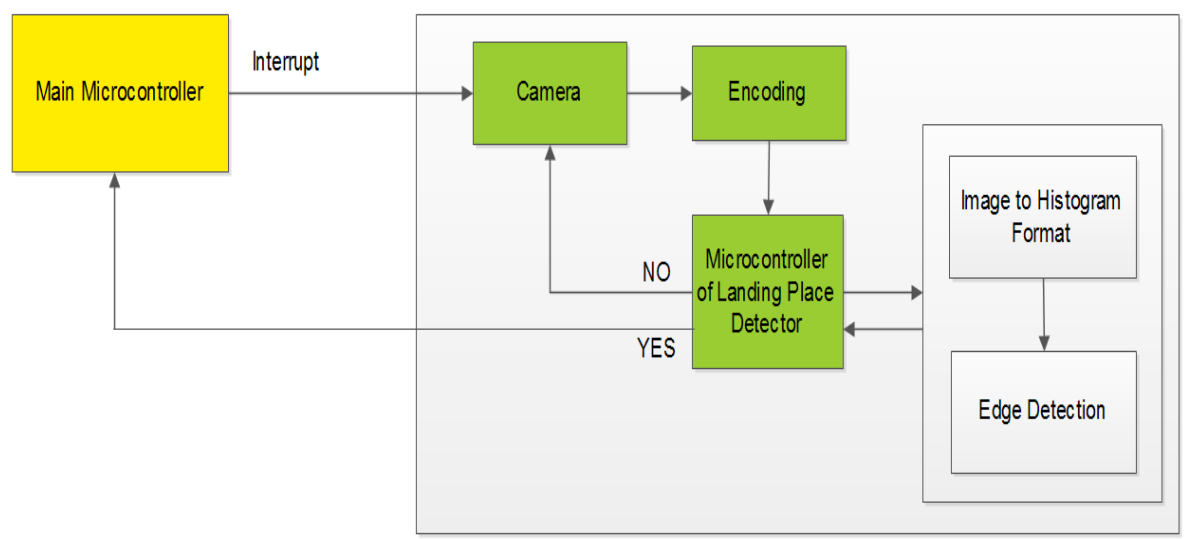

Figure 4. Block diagram of landing place detection in quadcopter

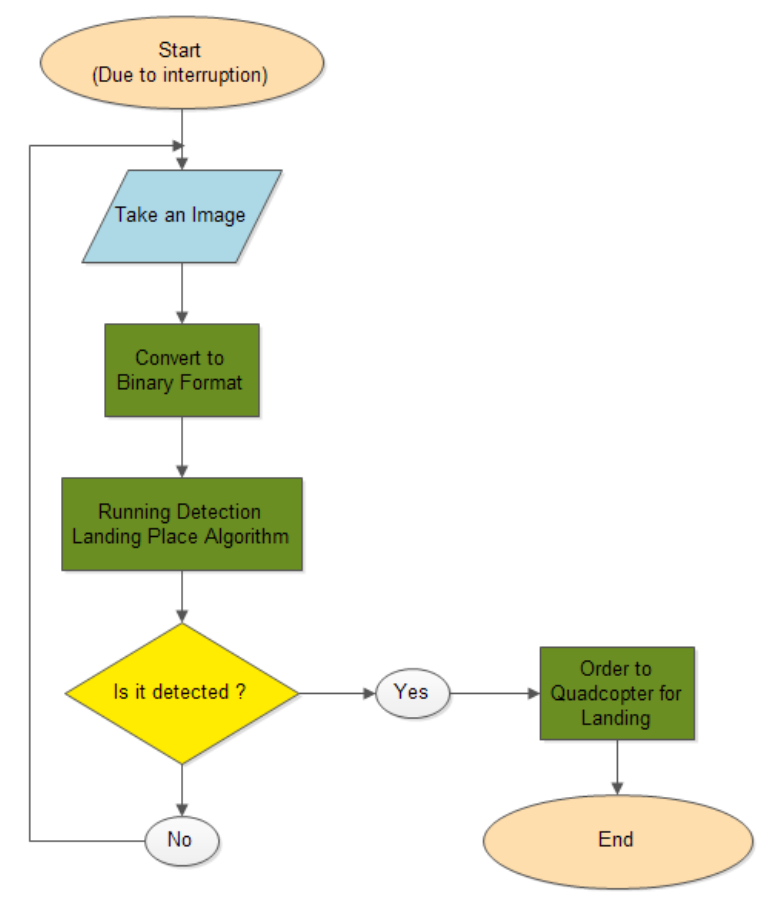

Figure 5. Implemented algorithm for detecting landing place of quadcopter

After interruption, which is sent out from main micro controller, the camera takes some frequent images and at the same time sends these to the encoding module for conversion into a binary format; Converted images sent into the landing place detector and the trace of shape will begin. Table 1 illustrates the list of modules and their methods which are employed in this paper. Those details will be presented in section below. 
Table 1. List of modules

\begin{tabular}{|l|l|l|l|}
\hline \multicolumn{1}{|c|}{ Module Name } & \multicolumn{1}{c|}{ Input } & \multicolumn{1}{c|}{ Output } & \multicolumn{1}{c|}{ Method } \\
\hline Main Microcontroller & Final Decision & Interrupt & $\begin{array}{l}\text { Dynamic } \\
\text { Programming } \\
\text { Dynamic } \\
\text { Programming }\end{array}$ \\
\hline Camera & & Order to Landing off & - \\
\hline Encoding & Take an image & Send to Encoding & $\begin{array}{l}\text { Dynamic } \\
\text { Programming }\end{array}$ \\
\hline Histogram & Image & Binary format of Image & Pattern Machine \\
\hline Edge Detection & Binary Image & Geometric Histograms & $\begin{array}{l}\text { Morphology } \\
\text { Operation }\end{array}$ \\
\hline
\end{tabular}

First of all, for checking the accuracy of detection and tracing, we considered our case in MATLAB software, after utilization and getting the satisfactory results, next was implementation. A suitable algorithm which was written in $\mathrm{C}++$ programming language was used for robotic programming and it creates understandable file that is programmed for SMD AVR micro controller. Figure 6 present our result in MATLAB software.

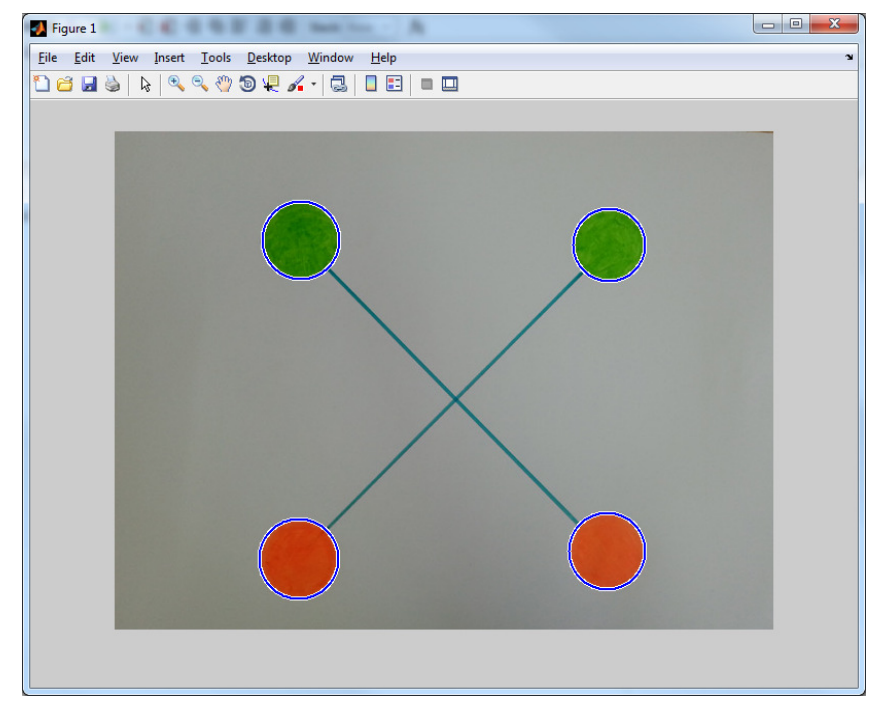

Figure 6. Result of detection circles which are defined for landing off quadcopter in MATLAB shapes.

\subsection{Main Microcontroller}

The micro controller which was used in this paper is in the same category of AVR family. Not only microcontroller has variety of duties on quadcopter, but also it keeps sending the interruption to camera module in order to catch our predefined image. The interruption which is determined for this case is Timer1 of micro controller. By using it, micro controller will be able to work in parallel mode. 


\subsection{Camera}

The camera module is essential for detection; obviously, it takes the image for detecting and tracing landing place and sends each of them to the next module, encoding module, the detecting process will begin.

\subsection{Encoding}

To employ image processing algorithm, the image should convert to a binary format. An image in binary format has only two possibilities for each pixel. Numerically, those values are 0 for black and 1 until 255 for white. This converter is so important for understanding the image processing algorithm which is used in this paper.

\subsection{Landing Place Detector}

The Histogram that is based on geometric histogram is valued in the horizontal and vertical directions; similarly it can be done by Euclidean distance. This sequence executes for each pixel in binary format to achieve the degree of vectors. For each histogram, degree of match between model feature $M j$ and image feature $I$ is calculated by:

$$
D_{J}=\sum_{i=1}^{n} \sqrt{I_{i} \cdot M_{j i}}
$$

Due to the geometric histogram of the binary format, another calculation would be the edge detection. The specific algorithm is employed to Euclidean distance in order to transform the image. First of all, for 2D images, the algorithm distinguishes the image resolution in $X$ and $Y$ directions and the edge pixels should be defined. Secondly, for each edge pixel there is an exact metric distance, so each pixel of the metric is correlated with a matching area of the binary image and they are assigned a distance value via Euclidean distance to get nearest pixels in binary format image. For two pixels point $P(x 1, y 1)$ and $Q(x 2, y 2)$ in 2D image the classical Euclidian distance is defined by:

$$
\sqrt{\left(x_{2}-x_{1}\right)^{2}+\left(y_{2}-y_{1}\right)^{2}}
$$

In order to extract the background distance via Euclidian distance formulation, the image resolution is utilized in $\mathrm{X}$ and $\mathrm{Y}$ dimensions, therefore:

$$
\sqrt{\left(x_{2}-x_{1}\right)^{2} \times x_{\text {resolution }}^{2}+\left(y_{2}-y_{1}\right)^{2} \times y_{\text {resolution }}^{2}}
$$

These formulas are applied for two areas in the image. Background and circular shapes in image which is taken from camera.

\section{EXPERIMENTS AND RESULTS}

Regarding to our proposed implementation methods in MATLAB software, to detect and trace circular shapes which are defined for landing place of quadcopter, we got good results about different images. So it is assumed that the quadcopter could carry out by this way. We have worked hard to ensure our proposition in experimental practice. See the experimental video clip on (https://www.youtube.com/watch?v=zfPzSbz93rE ). 


\section{CONCLUSIONS}

In this research paper, image processing technology based on edge detection was utilized to detect the landing spot of a quadcopter. Basically, the purpose of this study is to detect the landing place of homemade quadcopter, which has a great sensitivity in bright environments.

The hierarchical classifier that is implemented in this issue uses two distinct detectors. First one is the timer of main micro controller used to send interruptions to the camera. By frequent images that were taken via the camera, quadcopter can identify its predefined landing place. Meanwhile second detector has to apply the detection algorithm on the image to detect and trace the landing place. This method is examined by MATLAB software and satisfactory outcomes are achieved, and also this approach is very accurate and performs to detect landing place. However, its landing on exact place and recognition of the spot in foggy places are challenging issues and needs further improvements. It is believed that by this technique another step is taken toward autonomous flying robots.

\section{REFERENCES}

[1] Teppo Luukkonen, "Modelling and control of quadcopter," Independent research project in applied mathematics,Espoo, August 22, 2011.

[2] H. Huang, G. M. Hoffmann, S. L. Waslander, and C. J. Tomlin, "Aerodynamics and control of autonomous quadrotor helicopters in aggressive maneuvering," IEEE International Conference on Robotics and Automation, pp. 3277-3282, May 2009.

[3] B. Lavi, "HUMAN BODY DETECTION AND SAFETY CARE SYSTEM FOR A FLYING ROBOT", Second International Conference on Advanced Information Technologies and Applications (ICAITA), pp. 329-337, November 2013.

[4] Achtelik, M. C., Stumpf, J., Gurdan, D., \& Doth, K. M. (2011, September). Design of a flexible high performance quadcopter platform breaking the mav endurance record with laser power beaming. In Intelligent Robots and Systems (IROS), 2011 IEEE/RSJ International Conference on (pp. 5166-5172). IEEE.

[5] Kunzmann, J., \& Berry, C. A. (2011). Investigation in the Control of a Four-Rotor Aerial Robot.

[6] S. Bouabdallah, P. Murrieri, and R. Siegwart, Design and Control of an Indoor Micro Quadrotor, Proceedings of the 2004 IEEE International Conference on Robotics and Automation (ICRA), 5(26), New Orleans, LA, April 26 - May 1, 2004, 4393-4398.

[7] B. Erginer and E. Altug, Modeling and PD Control of a Quadrotor VTOL Vehicle", Proceedings of the 2007 IEEE Intelligent Vehicles Symposium (IV'07), Istanbul, Turkey, June 13-15, 2007, 894-899.

[8] S. Bouabdallah and R. Siegwart, Towards Intelligent Miniature Flying Robots, (Berlin, GE: Springer, 2006).

[9] P. Pounds, R. Mahony, P. Hynes, and J. Roberts, Design of a Four-Rotor Aerial Robot, Proceedings of the 2002 Australasian Conference on Robotics and Automation, Auckland, Australia, November 2 $-29,2002$.

[10] P. Pounds, R. Mahony, Corke, P. Corke, and J. Roberts, Towards Dynamically-Favourable QuadRotor Aerial Robots, Proceedings of the 2004 Australasian Conference on Robotics \& Automation (ARAA), Canberra Australia, December 6 - 8, 2004.

[11] A. Tayebi and S. McGilvray, Attitude Stabilization of a VTOL Quadrotor Aircraft, IEEE Transactions on Control Systems Technology, 14, May 2006, 562-571.

[12] González, I., Salazar, S., Torres, J., Lozano, R., \& Romero, H. (2013). Real-Time Attitude Stabilization of a Mini-UAV Quad-rotor Using Motor Speed Feedback. Journal of Intelligent \& Robotic Systems, 70(1-4), 93-106.

[13] A. Tayebi and S. McGilvray, "Attitude stabilization of a four-rotor aerial robot," 43rd IEEE Conference on Decision and Control, vol. 2, pp. 1216-1221,2004.

[14] Fatan,M.,Lavi,B.,Vatankhah,A.," An Adaptive Neuro PID for Controlling the Altitude of Quadcopter robot",18th international conference on methods and models in automation and robotics, August 2013

[15] X. Wang, T. Han, and S. Yan, "An HOG-LBP human detector with partial occlusion handling," in Proc. IEEE Int. Conf. Comput. Vis., 2009,pp. 32-39. 
[16] D. Geronimo, A. Sappa, A. Lopez and D. Ponsa, "Adaptive image sampling and windows classification for on-board pedestrian detection,"in Proc. 5th Int. Conf. Comput. Vis. Syst., 2007.

[17] Y. Pang, Y. Yuan, and X. Li, "Gabor-based region covariance matrices for face recognition," IEEE Trans. Circuits Syst. Video Technol., vol. 18,no. 7, pp. 989-993, Jul. 2008.

[18] O. Tuzel, F. Porikli, and P. Meer, "Pedestrian detection via classification on Riemannian manifolds," IEEE Trans. Pattern Anal. Mach. Intell.,vol. 30, no. 10, pp. 1713-1727, Oct. 2008.

[19] R. Lienhart and J. Maydt, "An extended set of Haar-like features for rapid object detection," in Proc. IEEE Int. Conf. Image Process., 2002, vol. 1,pp. 900-903.

[20] Lee, K. D., Nam, M. Y., Chung, K. Y., Lee, Y. H., \& Kang, U. G. (2013), "Context and profile based cascade classifier for efficient people detection and safety care system", Multimedia Tools and Applications, 1-18.

[21] Satake, J., Miura, J.: Robust Stereo-based Person Detectingand Tracking for a Person Following Robot. In:Proc. ICRA 2009 workshop on person detection and tracking. Kobe, Japan (2009)

[22] Wilhelm, T., Böhme, H.-J., Gross, H.-M.: A multimodal system for tracking and analyzing faces on a mobile robot. Robot. Auton. Syst. 48(1), 31-40, (2004),European Conference on Mobile Robots (ECMR '03)

[23] Medionia, G., R.J. Françoisa A., Siddiquia, M., Kima,K., Yoonb, H.: Robust real-time vision for a personal service robot. Comput. Vis. Image Understanding 108(1-2), 196-203, Special Issue on Vision for Human-Computer Interaction, October-November 2007

[24] Li, L., Koh, Y.T., Ge, S.S., Huang, W.: Stereo-based human detection for mobile service robots. Control, Automation, Robotics and Vision Conference, 2004, ICARCV, vol. 1, pp. 74-79 (2004)

[25] Bellotto, N., Hu, H.: Multisensor-based human detection and tracking for mobile service robots systems, man, and cybernetics, Part B: cybernetics. IEEE Trans.39(1), 167-181 (2009)

[26] Böhme, H.J., Wilhelma, T., Keya, J., Schauera, C., Schrötera, C.,Großa,H-M., Hempelb, T.:An approach to multi-modal human-machine interaction for intelligentservice robots. Robot. Auton. Syst. 44(1), 83-96(2003)

[27] Meis, U., Oberlander, M., Ritter, W.: Reinforcing the reliability of pedestrian detection in far-infrared sensing. 2004 IEEE Intelligent Vehicles Symposium,pp. 779-783, 14-17 June 2004

[28] Mudaly, S.S.: Novel computer-based infrared pedestrian data-acquisition system. Electron. Lett. 15(13), 371-372 (1979)

[29] Nanda, H., Davis, L.: Probabilistic template based pedestrian detection in infrared videos. IEEE Intell. Veh. Symposium 1, 15-20 (2002)

[30] Bertozzi, M., Broggi, A., Fascioli, A., Graf, T., Meinecke, M.-M.: Pedestrian detection for driver assistance using multiresolution infrared vision. IEEE Trans. Veh. Technol. 53(6), 1666-1678 (2004)

\section{AUTHORS}

Bahram Lavi Sefidgari received a B.S degree from Islamic Azad University of Tabriz, Iran. Currently, $\mathrm{He}$ is a post graduate student in Computer Engineering in Eastern Mediterranean University (EMU) and work in computer center of university. His research interest is Artificial Intelligent in robotics.

Sahand Pourhassan Shamchi received a B.S degree from Islamic Azad University of Tabriz, Iran. Currently, He is a post graduate student in Mechanical Engineering in Eastern Mediterranean University (EMU). His research interest is manufacturing.
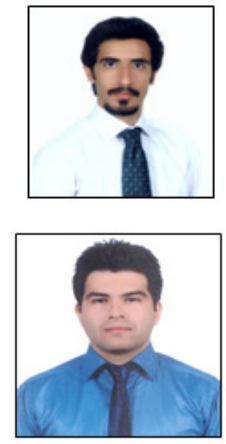